\title{
Feeling we're biased: Autonomic arousal and reasoning conflict
}

\author{
Wim De NeYs \\ CNRS and University of Toulouse, Toulouse, France \\ AND \\ Elke Moyens and Debora Vansteenwegen \\ University of Leuven, Leuven, Belgium
}

\begin{abstract}
Human reasoning is often biased by intuitive beliefs. A key question is whether the bias results from a failure to detect that the intuitions conflict with logical considerations or from a failure to discard these tempting intuitions. The present study addressed this unresolved debate by focusing on conflict-related autonomic nervous system modulation during biased reasoning. Participants' skin conductance responses (SCRs) were monitored while they solved classic syllogisms in which a cued intuitive response could be inconsistent or consistent with the logical correct response. Results indicated that all reasoners showed increased SCRs when solving the inconsistent conflict problems. Experiment 2 validated that this autonomic arousal boost was absent when people were not engaged in an active reasoning task. The presence of a clear autonomic conflict response during reasoning lends credence to the idea that reasoners have a "gut" feeling that signals that their intuitive response is not logically warranted. Supplemental materials for this article may be downloaded from http://cabn.psychonomic -journals.org/content/supplemental.
\end{abstract}

In the spring of 2009, fears of the H1N1 virus swept the world. The media commonly referred to the new virus as "swine" or "Mexican" flu although it was no longer harbored in swine and had already spread over the world at the time of the outbreak; hence, eating pork or having dinner at your local Mexican restaurant did not pose any clear health risks. The World Health Organization tried hard to inform the public, but the mere intuitive association with the name of the virus seemed to have an irresistible pull on people's behavior: A lot of us stopped eating at Mexican restaurants, Haitian officials rejected an aid ship with Mexican food aid, pork belly futures collapsed on Wall Street, and the Egyptian government even ordered their farmers to kill all of their pigs (Alexander, 2009; Ballantyne, 2009). From a logical point of view, none of these measures was effective to stop the spread of the virus or avoid contamination, but, intuitively, people nevertheless felt they were better off by simply avoiding contact with Mexicans or pork.

People's overreaction to the swine flu threat is a dramatic illustration of a general human tendency to base our judgment on fast intuitive impressions rather than on more demanding, deliberative reasoning. This tendency is biasing people's performance in a wide range of classic logical and probabilistic reasoning tasks (Evans, 2003; Kahneman, 2002). One of the most famous and studied examples is the belief bias phenomenon in syllogistic rea- soning. Belief bias refers to the intuitive tendency to judge the validity of a syllogism by evaluating the believability of the conclusion (Oakhill, Johnson-Laird, \& Garnham, 1989). Often this is problematic, because the believability of the conclusion conflicts with its logical status. Consider the following example: "All birds have wings. Crows have wings. Therefore, crows are birds." Although the conclusion in the example is logically invalid and should be rejected, intuitively many people will nevertheless tend to accept it because it fits with their prior beliefs. Sound reasoning requires that people abandon this mere intuitive, or so-called "heuristic," thinking, and engage in more deliberate, analytic thinking. Unfortunately, this turns out to be quite hard for most people; just as in the swine flu case, many reasoners end up being biased by their intuition.

Although it is a well-established fact that people are often biased, the nature of this bias is unclear. The crucial issue boils down to whether or not people detect that they are biased. Sound reasoning requires that people monitor their intuitions for conflict with more logical considerations. According to one view, people would be very bad at this monitoring (e.g., Kahneman \& Frederick, 2005). Because of lax monitoring, people would simply not detect that their intuitions are invalid. However, others have argued that there is nothing wrong with the detection process (e.g., Epstein, 1994; Houdé, 2007; Sloman, 1996). They claim that people have little trouble detecting that 
their intuitions are not fully warranted; the problem, according to this view, is that these intuitions are so tempting that people fail to discard them.

Clarifying the efficiency of the conflict detection process and the resulting nature of the heuristic bias is crucial for the study of human thinking. Recently, De Neys, Vartanian, and Goel (2008) tried to decide between the alternative views by monitoring the activation of the anterior cingulate cortex (ACC), a neural region associated with conflict detection, during reasoning. They observed that the neural conflict region was activated when people gave biased responses. This finding provided some preliminary support for the idea that people detect that they are biased. However, settling the debate requires further validation and characterization of the detection process. The present study addresses this issue by focusing on autonomic nervous system modulation during biased reasoning.

The inspiration for this study came from basic cognitive control studies (e.g., Botvinick, Cohen, \& Carter, 2004; Ridderinkhof, Ullsperger, Crone, \& Nieuwenhuis, 2004). In these studies people are typically presented with elementary conflict tasks in which they need to withhold an inappropriate but dominant response. Previous work in this field showed that the ACC is especially sensitive to the presence of conflict between competing responses (e.g., van Veen \& Carter, 2006). The initial study of De Neys et al. (2008) established that this same cortical conflict region was activated when people gave biased responses in a classic reasoning task. However, more recently it has been shown in the cognitive control field that, besides ACC activation, the elementary conflicts also elicit global autonomic arousal (Kobayashi, Yoshino, Takahashi, \& Nomura, 2007). In other words, at least in the elementary control tasks, the presence of conflict seems to be accompanied by visceral arousal, as reflected, for example, in increased skin conductance (Hajcak, McDonald, \& Simons, 2003). This suggests that basic measures of electrodermal activation can be used as a biological index of conflict detection in the reasoning field. On the basis of the cognitive control findings, one may expect that if conflict detection during thinking is indeed flawless, solving reasoning tasks in which intuitions conflict with logic will elicit increased skin conductance response (SCR).

In the present study, we tested this idea by monitoring participants' SCR while they were solving the infamous syllogistic reasoning problems. For half of the presented problems, referred to as conflict problems, the believability of the conclusion conflicted with its logical status, such that, just as in the introductory example, mere intuitive and logical thinking cued an inconsistent response. The other half of the problems were control or no-conflict problems, in which the believability of the conclusion was consistent with its logical status (e.g., a valid syllogism with a believable conclusion). Consider the following example: "All birds have wings. Crows are birds. Therefore, crows have wings." Both a priori beliefs and logical considerations will tell participants to accept the conclusion. In this case there is no conflict and no need to discard the intuitive beliefs.

Contrasting the SCR while people are solving conflict and no-conflict problems will allow us to settle the bias debate. If the bias-as-inhibition-failure view is right, and people realize that their intuition conflicts with the logical appropriate response, the resulting conflict should elicit autonomic arousal, which should be reflected in increased SCRs for the conflict (vs. no-conflict) problems. However, if the conflict monitoring is lax, and people do not detect the inherent intuition-logic clash on the conflict problems, autonomic arousal levels should not differ for the conflict and no-conflict problems.

We clarified that the present study will provide a much needed test of the initial conflict detection findings. However, examining a possible autonomic conflict response also has important conceptual implications. Note that it has long been known that people's online verbalizations during thinking, and their retrospective response justifications, typically do not suggest that they are taking any logical considerations into account (e.g., Evans \& Over, 1996; Wason \& Evans, 1975); however, although there might be some initial empirical findings suggesting that people detect the presence of an intuition-logic conflict, it is also pretty clear that people do not express this explicitly (e.g., De Neys \& Glumicic, 2008). Therefore, it has been hypothesized that conflict detection during thinking is a quite implicit process. Franssens and De Neys (2009; see also Thompson, 2009) suggested that it should be conceived as a "gut feeling": People would experience some general arousal resulting from the conflict detection, but they would not manage to label the detected logical violations explicitly. Bluntly put, people would sense that their response was wrong, but they would not manage to put their finger on it and explain verbally why their response is wrong. However, this post hoc characterization has not been tested directly. Establishing a possible link between autonomic modulation and the conflict detection might help to provide more solid conceptual ground for the idea that people literally "feel" the presence of conflict between their intuitions and logical considerations.

\section{EXPERIMENT 1}

\section{Method}

Participants. Thirty University of Leuven undergraduates who had not taken logic courses participated in return for a small monetary reimbursement.

Materials. The syllogistic reasoning task was based on the work of Sá, West, and Stanovich (1999). Participants evaluated eight conditional syllogisms. Four of the problems had conclusions in which logic was in conflict with believability (i.e., conflict problems: two problems with an unbelievable-valid conclusion, and two problems with a believable-invalid conclusion). For the other four problems, the believability of the conclusion was consistent with its logical status (i.e., no-conflict problems, two problems with an unbelievableinvalid conclusion, and two problems with a believable-valid conclusion). Each problem consisted of a major premise, minor premise, and conclusion. The following item format was adopted (note that the technical labels in italics were not presented on screen):

Major premise: $\quad$ All fruits can be eaten
Minor premise: Hamburgers can be eaten
$\begin{aligned} & \text { Conclusion: } \\ & \text { Response alternatives: a. Conclusion follows logically }\end{aligned}$

b. Conclusion does NOT follow logically 
Table 1

Reasoning Accuracy (Percentage Correct $[\mathrm{PC}]$ ) and Response Times (RTs, in Milliseconds) in the Different Experiments

\begin{tabular}{|c|c|c|c|c|c|c|c|c|}
\hline \multirow[b]{3}{*}{ Task } & \multicolumn{4}{|c|}{ Accuracy } & \multicolumn{4}{|c|}{ RTs } \\
\hline & \multicolumn{2}{|c|}{ Conflict } & \multicolumn{2}{|c|}{ No Conflict } & \multicolumn{2}{|c|}{ Conflict } & \multicolumn{2}{|c|}{ No Conflict } \\
\hline & $\mathrm{PC}$ & $S E$ & $\mathrm{PC}$ & $S E$ & RT & $S E$ & RT & $S E$ \\
\hline Experiment 1: Reasoning & 52 & 6.0 & 89 & 2.7 & 4,033 & 339 & 3,314 & 232 \\
\hline Experiment 2: Belief task & 24 & 6.1 & 95 & 2.8 & 3,151 & 305 & 2,504 & 224 \\
\hline
\end{tabular}

Note-For comparison, we refer to the logical response as the correct response in Experiment 2.

To minimize the possibility that the content of the conflict and noconflict problems affected the results, we constructed two problem sets in which the content was crossed (see the supplemental materials, Table S1). Each set was presented to half of the participants. The premise and conclusion believability of the conflict and no-conflict problems in each set was matched.

Each trial lasted 18,500 msec. First, a fixation cross was presented for $1,000 \mathrm{msec}$. Then, the major premise was presented for $3,000 \mathrm{msec}$. Next, the minor premise was presented for 2,000 msec. Finally, the conclusion and response options were presented. The complete problem remained on the screen for another $10,500 \mathrm{msec}$. After the $10,500 \mathrm{msec}$ had elapsed, the screen was cleared, and after an additional 2,500-msec rest interval, the next problem was presented.

Participants received standard deductive reasoning instructions that stressed that the premises should be assumed to be true, and that a conclusion should be accepted only if it followed logically from the premises. Before the start of the experiment, participants were familiarized with the presentation format and shown one example item.

Skin conductance recording. Standard $\mathrm{Ag} / \mathrm{AgCl}$ electrodes (1-cm diameter) filled with a Unibase electrolyte were attached to the hypothenar palm of each participant's nondominant hand. The interelectrode distance was $2.5 \mathrm{~cm}$. A Coulbourn skin-conductance coupler (V71-23) provided a constant $0.5 \mathrm{~V}$ across electrodes. The analogue conductance signal was passed through a 12-bit AD converter and digitized at $10 \mathrm{~Hz}$. The resulting skin conductance signal was visually inspected, corrected for artifacts, and retained for analysis.

The SCR was quantified by a difference score between the maximum and the minimum skin conductance value within our time intervals of interest (e.g., Botvinick \& Rosen, 2009; Dawson, Schell, \& Filion, 2000). ${ }^{1}$ Detection of a conflict between conclusion believability and validity can occur only after presentation of the conclusion and can be expected to be processed by the time a response has been given. Therefore, our main focus was the SCR in the interval between the presentation of the conclusion and the participant's response keypress; we refer to this interval as the reasoning phase. For completeness, we also looked at the SCR in the postresponse window between the response keypress and the end of the trial (postresponse phase), and in the interval between the start of the trial and the presentation of the minor premise (reading phase). Unless noted otherwise, however, all reported analyses concern the crucial reasoning phase.

\section{Results and Discussion}

Behavioral reasoning performance. Participants' performance on the reasoning task was as expected. People were typically biased when cued beliefs and logic conflicted, but had significantly less trouble in solving the no-conflict problems. Overall, correct response rates reached $52 \%$ on the conflict problems and $89 \%$ on the noconflict problems $\left[F(1,29)=27.38, p<.0001, \eta_{\mathrm{p}}^{2}=.49\right]$. As Table 1 shows, no-conflict problems were also solved faster than conflict problems were $[F(1,29)=8.18, p<$ $\left..01, \eta_{\mathrm{p}}^{2}=.22\right]$. These results closely replicate the findings in previous studies with similar syllogistic reasoning problems (e.g., De Neys, 2006; Markovits \& Nantel, 1989).
SCR. The main question was whether, despite the bad reasoning performance on the conflict problems, people would nevertheless detect that their intuitive response was not warranted and show increased autonomic arousal when solving these problems. As Figure 1 indicates, results showed that the SCRs in the crucial reasoning phase were significantly higher when conflict problems rather than no-conflict problems were being solved $[F(1,29)=5.70$, $\left.p<.025, \eta_{\mathrm{p}}^{2}=.16\right]$. SCRs for the conflict and no-conflict problems did not differ, however, in the initial reading phase $[F(1,29)=1.87, p=.18]$ and in the postresponse phase $[F(1,29)<1]$. Hence - consistent with the idea that the autonomic arousal results from conflict detectionSCRs increased only after the conflict was introduced, and leveled off after participants had responded near the end of the trial. This pattern indicates that the observed increased autonomic arousal is specifically tied to conflict detection.

Note that our time window of interest for the reasoning phase was the interval between presentation of the conclusion and the participant's response keypress. The behavioral data indicated that with an average response time (RT) of about 4,000 msec, participants needed more than $700 \mathrm{msec}$ longer to solve the conflict problems than to solve the no-conflict ones. This implies that the reasoning phase was typically longer for the conflict trials. One might argue that it is the longer RT per se that drives the observed higher SCRs in the reasoning phase. To eliminate such a confound, we ran an additional analysis in which we controlled the length of the reasoning interval on the conflict trials. Only the skin conductance values during the first 3,000 msec after conclusion presentation were taken into account (i.e., a cutoff value about one $S E$ below the average RT for the no-conflict problems). Hence, in this control analysis, the reasoning phase was actually slightly shorter for the conflict problems than for the no-conflict ones; however, the overall pattern of results was not affected. The SCRs were still significantly higher when conflict problems rather than no-conflict problems were being solved $\left[F(1,29)=4.64, p<.05, \eta_{\mathrm{p}}^{2}=.14\right]^{2}$ This establishes that the longer conflict latencies in our standard analysis do not drive the higher SCRs.

The overall results support the idea that conflict detection during thinking is associated with increased autonomic arousal, but they do not yet allow us to decide between the lax- and flawless-detection views. Although reasoning accuracy on the conflict problems was low, some people did manage to respond correctly. Both the lax and flawless views entail that good reasoners, who 


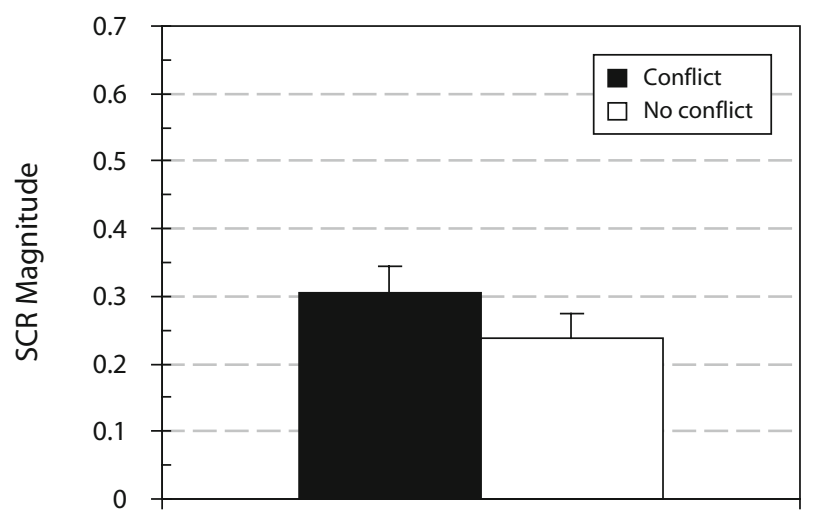

Reasoning

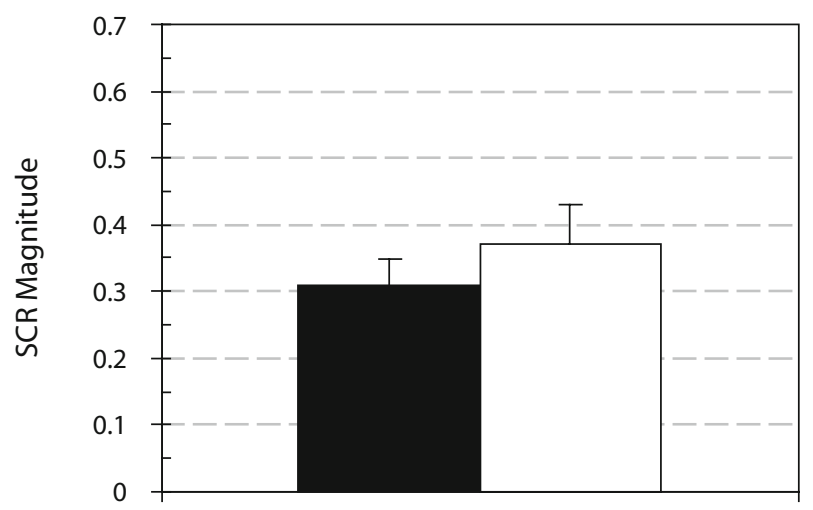

Initial Reading

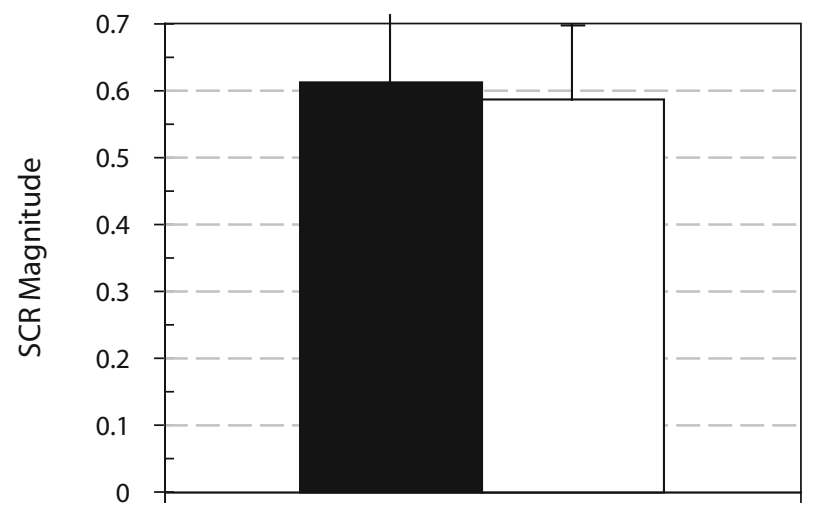

Postresponse

Figure 1. Average skin conductance response (SCR) magnitude (in $\mu \mathrm{S}$ ) for conflict and no-conflict syllogisms during reasoning interval (top panel), initial reading (middle panel), and postresponse (bottom panel). Error bars indicate standard errors.

manage to solve the problems correctly, will detect the unwarranted nature of the intuitive response. Hence, although overall accuracy was low, a supporter of the laxdetection view could still argue that the observed overall SCR increase was simply driven by the good reasoners. To address this critique, we looked at the association be- tween accuracy on the conflict problems and the extent of the observed SCR increase on these problems during the reasoning phase.

If only good reasoners were to show the increased arousal, there should be a clear link between the two factors. However, a correlation analysis showed that the SCR increase (i.e., SCR conflict problems - SCR no-conflict problems) did not depend on reasoning accuracy $(r=.15$, $p=.413$ ). To explore this issue further, Figure 2 shows the SCRs during the reasoning phase for good and bad reasoners on the basis of a median split on the conflict accuracy. Average reasoning accuracy in the good group $(n=$ 13) was $89 \%$ and $24 \%$ in the bad group $(n=17)$. As Figure 2 illustrates, the crucial SCR increase on the conflict problems did not depend on the skill factor $[F(1,28)<1]$. Overall, good reasoners did tend to have higher SCRs but this trend was not significant $[F(1,28)<1]$.

In an additional analysis, we looked at even more extreme skill groups. We contrasted performance of the very best and worst reasoners in our sample; people who solved none $(n=6)$ or all $(n=7)$ of the conflict problems correctly. Overall, SCRs were still significantly greater for conflict than for no-conflict problems $[F(1,11)=$ $6.77, p<.025, \eta_{\mathrm{p}}^{2}=.38$ ], but even for these extreme groups the increase did not differ for good and bad reasoners $[F(1,11)=2.10, p=.17]$. These findings clearly establish that the observed overall SCR increase is not solely driven by the good reasoners. Consistent with the flawless-detection view, everybody seems to be detecting the conflict between cued intuitions and the logical appropriate response.

A last issue we need to address is the impact of possible learning effects. The initial studies that started focusing on conflict detection were typically quite lengthy. For example, in their fMRI study De Neys et al. (2008) presented almost 100 reasoning items. One might argue that the repeated presentation and repetitive nature of these studies cued participants to start paying attention

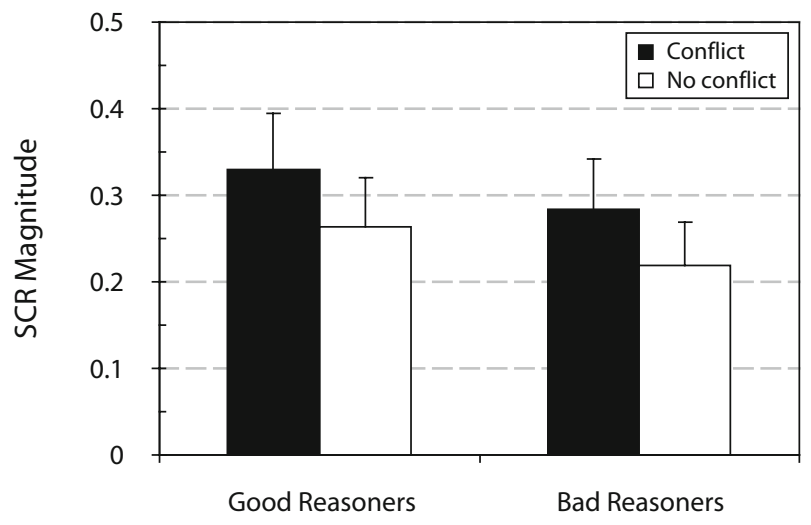

Reasoning Skill

Figure 2. Average skin conductance response (SCR) magnitude (in $\mu \mathrm{S}$ ) during the reasoning interval for conflict and no-conflict syllogisms of the best (good reasoners) and worst (bad reasoners) scoring half of the participants. Error bars indicate standard errors. 


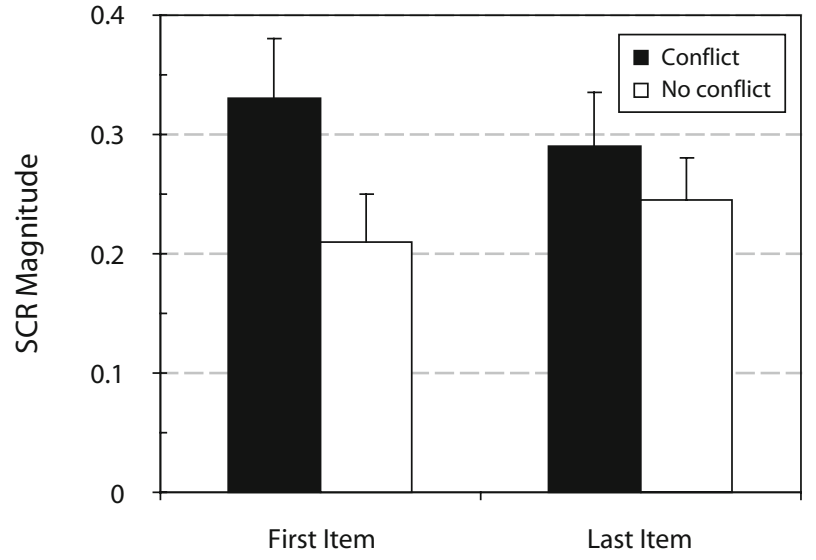

Figure 3. Average skin conductance response (SCR) magnitude (in $\mu \mathrm{S}$ ) during the reasoning interval for the first and last presented conflict and no-conflict syllogisms. Error bars indicate standard errors.

to the conflict manipulation; hence, the flawless detection in these studies might simply be an artifact that results from a learning effect. Note that we already reduced the number of presented items in the present study to limit the impact of such a learning confound. However, to address the issue further, we repeated our analysis solely with the first presented conflict and no-conflict problem that every participant solved.

Contrary to the learning hypothesis, we replicated the overall pattern. As Figure 3 shows, right from the start of the experiment SCRs were higher for conflict than for no-conflict problems $\left[F(1,29)=6.61, p<.025, \eta_{\mathrm{p}}^{2}=\right.$ .19]. Note that, as with the overall analysis, the increase did not depend on whether participants were good or bad reasoners and managed to solve the conflict problem correctly $\left(r_{\mathrm{s}}=.06, p=.75\right)$. We also contrasted the SCRs for the last conflict and no-conflict problem that participants solved. As Figure 3 shows, the SCR increase on the conflict problems tended to become less pronounced near the end of the experiment. Although this trend was not significant $[F(1,29)=1.43, p=.24]$, it clearly argues against the learning hypothesis. If anything, repeated presentation tended to result in autonomic habituation and decreased rather than boosted the observed effects.

\section{EXPERIMENT 2}

Experiment 1 established that dealing with conflicting logical and tempting intuitive responses during reasoning resulted in increased autonomic arousal, as reflected in increased SCRs. This visceral conflict response did not result from a learning effect and was shown by all reasoners. Thereby, the findings are consistent with the view that reasoning bias cannot be attributed to a conflict detection failure. However, the apparent omnipresence of the arousal signal also gives rise to possible alternative accounts. It could be argued, for example, that the increased SCRs do not result from a reasoning-related detection process per se, but simply from a superficial mate- rial characteristic or a more general process not related to reasoning. Although our study design minimized such a possible confound, it cannot be completely discarded. In a second control experiment, we therefore addressed this issue directly.

In Experiment 2, we presented participants the exact same material as in Experiment 1, but simply asked them to evaluate the believability of the conclusion. Hence, in this task there was no need to engage in a logical reasoning process. Consequently, since the task will not cue a logical response, there should also not arise a conflict between a cued logical and intuitive response; so if it is really the case that the observed increased SCRs in Experiment 1 result from a reasoning-related conflict detection process, we should no longer observe them in Experiment 2.

\section{Method}

Participants. Thirty University of Leuven undergraduates who had not taken logic courses participated in return for a small monetary reimbursement. The data of Experiment 1 were used as a baseline to test the impact of the task manipulation.

Materials. In the belief evaluation task, participants were presented with the same items as in Experiment 1. The task was introduced to participants as a pilot study in which the believability of a number of statements needed to be evaluated. Any references to logical reasoning in the task instructions were avoided. Participants were told that they would see short stories consisting of three sentences and that they simply needed to indicate whether they believed the final sentence or not. The two response alternatives were rephrased as " 1 . The sentence is believable" and " 2 . The sentence is not believable." Instructions stressed "it was fine to select the response that came first to mind and seemed intuitively most plausible." Previous studies indicated that some participants spontaneously engage in logical reasoning when presented with conditional syllogisms, even when they are not explicitly instructed to do so (e.g., De Neys, Schaeken, \& d'Ydewalle, 2005). The present task modifications minimized such a possible confound. Except for these modifications, the presentation procedure was identical to Experiment 1.

In the skin conductance recording, the same procedure as in Experiment 1 was used. Data of 1 participant were lost due to equipment failure and were not included in the analyses.

\section{Results and Discussion}

Behavioral performance. Accuracy and response latencies established that the task manipulation was successful. As Table 1 indicates, participants gave overall more belief-based responses, when instructed to do this in Experiment 2, than in Experiment $1[F(1,57)=5.44, p<$ $\left..025, \eta_{\mathrm{p}}^{2}=.09\right]$. This tendency was more pronounced on the conflict than on the no-conflict problems $[F(1,57)=$ $\left.12.38, p<.001, \eta_{\mathrm{p}}^{2}=.18\right]$. Overall, responses were also given faster in the belief evaluation task $[F(1,57)=6.32$, $\left.p<.025, \eta_{\mathrm{p}}^{2}=.15\right]$. These faster responses were equally clear for conflict and no-conflict problems $[F(1,57)<1]$. The trend toward faster and more frequent belief-based responses indicates that participants indeed engaged in a more intuitive mode of processing in Experiment 2; this demonstrates that our instruction manipulation was successful.

SCRs. Figure 4 shows the average SCRs after conclusion presentation for conflict and no-conflict problems in the reasoning and belief evaluation task. An ANOVA established that the impact of the conflict factor clearly 
differed in both tasks $\left[F(1,57)=4.65, p<.05, \eta_{\mathrm{p}}^{2}=.08\right]$. As Figure 3 shows, SCRs were overall lower in the belief evaluation task $\left[F(1,57)=8.41, p<.01, \eta_{\mathrm{p}}^{2}=.13\right]$, but contrary to Experiment 1, SCRs for conflict and no-conflict problems no longer differed in the belief evaluation task $[F(1,57)<1]$. Hence, when people were not engaged in reasoning, and cued intuitions did not conflict with logical considerations, autonomic arousal did not increase when conflict problems were being solved. This finding establishes that the SCR increase we observed in Experiment 1 results from reasoning-related conflict detection.

SCR and believability $\times$ validity interaction. The believability and validity of the problems in our study were completely crossed. We were interested in the overall conflict between these two factors, and had no specific hypotheses about possible further lower level interactions. As in previous studies, our analyses therefore focused on the main conflict factor and collapsed data over the belief and validity levels (e.g., De Neys, 2006; Goel \& Dolan, 2003; Stanovich \& West, 2000; Tsujii \& Watanabe, 2009). For completeness, we did enter the two factors separately in a 2 (believability) $\times 2$ (validity) $\times 2$ (experiment) mixed-model ANOVA on the SCRs in the reasoning phase. Table 2 gives an overview of the findings. There were main effects of the believability and experiment factors: Overall SCRs were lower in Experiment 2 than in Experiment $1\left[F(1,57)=8.57, p<.005, \eta_{\mathrm{p}}^{2}=.13\right]$, and higher for unbelievable than for unbelievable conclusions $\left[F(1,57)=4.99, p<.05, \eta_{\mathrm{p}}^{2}=.08\right]$. The interaction between the three factors was also marginally significant $\left[F(1,57)=3.70, p<.06, \eta_{\mathrm{p}}^{2}=.06\right]$. Other effects and interactions failed to reach (marginal) significance. We explored the three-way interaction with planned contrast. For the reasoning task in Experiment 1, results showed that there was a significant believability $\times$ validity interaction $\left[F(1,57)=6.58, p<.025, \eta_{\mathrm{p}}^{2}=.10\right]$. For the belief evaluation task in Experiment 2, this interaction was not significant $[F(1,57)<1]$. Further planned contrasts on

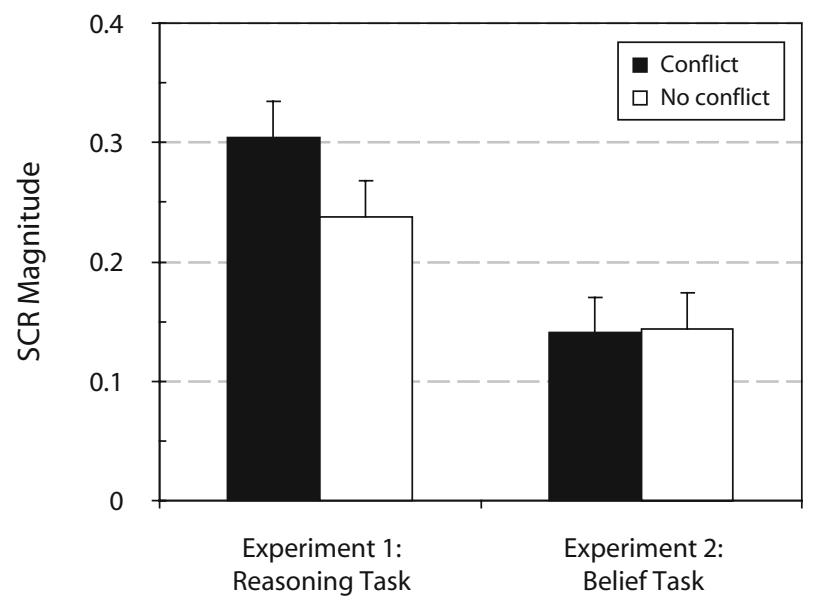

Figure 4. Average skin conductance response (SCR) magnitude (in $\mu \mathrm{S}$ ) during the reasoning interval for conflict and no-conflict syllogisms in Experiments 1 and 2. Error bars indicate standard errors.
Table 2

Average Skin Conductance Response (in $\mu \mathrm{S}$ ) During the Reasoning Interval As a Function of Conclusion Validity and Believability in Experiments 1 and 2

\begin{tabular}{lllll}
\hline & \multicolumn{4}{c}{ Believability } \\
\cline { 2 - 3 } \multicolumn{1}{c}{ Validity } & \multicolumn{2}{c}{ Believable } & & \multicolumn{2}{c}{ Unbelievable } \\
\cline { 2 - 3 } \cline { 5 - 6 } & $M$ & $S E$ & & $S E$ \\
\hline Experiment 1: Reasoning & .19 & .03 & .30 & .04 \\
$\quad$ Valid & .31 & .03 & .30 & .05 \\
$\quad$ Invalid & & & & .04 \\
Experiment 2: Belief task & .11 & .03 & .17 & .04 \\
$\quad$ Valid & .11 & .03 & .17 & .05 \\
$\quad$ Invalid & &
\end{tabular}

the Experiment 1 data indicated that the effect of validity was significant for the believable conclusions $[F(1,57)=$ 14.23, $\left.p<.001, \eta_{\mathrm{p}}^{2}=.20\right]$, but not for the unbelievable ones $[F(1,57)<1]$. This implies that the global conflict effect is especially driven by the believable problems. Being presented with unbelievable material seems to lead to a general SCR boost that blurs an additional effect of logical validity. However, on the believable problems it was still the case that SCRs were higher when the conclusion was logically invalid and beliefs and logic conflicted. Consistent with our hypothesis, this conflict-related SCR increase on the believable problems was completely absent when people were simply evaluating the conclusion believability in Experiment $2[F(1,57)<1]$.

\section{GENERAL DISCUSSION}

The present study shows that dealing with conflicting logical and intuitive responses during reasoning is accompanied by an increase in autonomic arousal. The increased autonomic arousal was reflected in an SCR boost right after the erroneous intuition was cued in conflict syllogisms but was absent when people were not engaged in a reasoning task. This establishes that the autonomic arousal is specifically tied to the detection of the conflict between logic and intuition. The finding that even the most biased reasoners showed the autonomic conflict response validated the view that intuitive bias cannot be attributed to a detection failure. Although not everyone might manage to discard the tempting intuitive response, all reasoners seem to be sensitive to the presence of the conflict.

The presence of a clear autonomic conflict response lends credence to the idea that conflict detection can be conceived as a "gut feeling" (e.g., Franssens \& De Neys, 2009; see also Thompson, 2009, for related suggestions). People seem to detect that their intuitions conflict with more logical considerations at the visceral level. At this point the present conflict findings show an interesting link with the seminal work of Bechara, Damasio, and colleagues (e.g., Bechara, Damasio, Tranel, \& Damasio, 1997; Damasio, 1994). In Bechara and Damasio's studies, participants were presented with a gambling task in which they could select cards from decks with different payoffs. Bechara and Damasio observed that participants needed about 80 trials before they could explicitly point out which decks were the good and bad ones. However, 
when the authors examined people's autonomic responses, they noticed that after a number of trials (i.e., between the 10th and 50th trial) people started showing an SCR increase (i.e., a "somatic marker") before they selected a card from the bad decks. Hence, although people had not yet acquired explicit knowledge of which decks were the bad ones, their autonomic response indicated that they implicitly acquired this knowledge. It will be evident that both Bechara and Damasio's work and the present study share the general idea that there is "more than meets the eye" in human thinking: Although people's overt response (i.e., their answer on the reasoning tasks) might be biased, this does not imply that they cannot detect this bias at a more implicit level. However, at the same time, we would like to point out that the autonomic signal that Bechara and Damasio focused on is different from the conflictdriven autonomic arousal signal that we report. First, Bechara and Damasio argued that the autonomic reaction in their gambling task resulted from the negative feedback (i.e., losing money) participants received after selecting bad cards. Clearly, in our classic reasoning task, such performance feedback was completely absent. Furthermore, our autonomic conflict response was present right from the start and did not result from a learning effect. Lastly, Bechara and Damasio observed their somatic markers only before participants made erroneous selections. As one would expect from a conflict-related detection signal, we observed our increased SCRs for both bad and good responses. Hence, although our findings and Bechara and Damasio's work both point to the importance of the autonomic response level in human thinking, the two bodies of work seem to deal with different autonomic signals.

The crucial implication of the present study is that people are less ignorant than the widespread intuitive bias suggests. Although people might not manage to label the logical principles being violated, the presence of the autonomic conflict response establishes that logical considerations are implicitly taken into account during reasoning. This implies that humans are no mere intuitive, illogical reasoners who disregard the logical implications of their judgments. Although our inferences are often biased, we do seem to sense that we are wrong.

We believe that our findings nicely illustrate the relevance of the cognitive neuroscience literature on cognitive control for the reasoning field (e.g., Botvinick et al., 2004; Ridderinkhof et al., 2004). The neuroscience toolbox allows reasoning researchers to start scratching below the response surface and address the key theoretical debates. In our view, a further continuation of the crosstalk between the fields might prove especially fruitful. For example, one interesting line for further study is the link between the presently observed autonomic response and previously established ACC activation during biased reasoning (De Neys et al., 2008). At least with the elementary cognitive control tasks, it has been suggested that the autonomic conflict signal originates in the ACC (e.g., Critchley, Tang, Glaser, Butterworth, \& Dolan, 2005; Hajcak et al., 2003). Combining fMRI and SCR recordings during reasoning might allow us to examine how strongly both signals covary, and whether one or the other specific subdivision within the ACC (e.g., dorsal or rostral ACC) drives the autonomic signal. Such research could also help to further distinguish the presently observed autonomic conflict signal from Bechara and Damasio's somatic marker signals (which have been shown to originate from the ventromedial prefrontal cortex; e.g., Bechara et al., 1997). Clearly, looking at the reasoning performance and SCRs of patients with lesions in the identified brain areas could then be used to further specify the neural underpinning of the autonomic conflict signal.

In the present study, we focused on popular syllogistic reasoning problems to study the conflict between intuitive and logical thinking. As we noted in the introduction, such conflicts arise in a wide range of classic reasoning and decision-making tasks. Clearly, it would be worthwhile to test the generalizability of the present findings across these tasks. Such generalization might be especially interesting, given the possible distortion that seemed to be created by the presence of unbelievable material in the present study. We mentioned that, when working with syllogisms, one typically creates conflict by crossing the problem believability with logical validity (e.g., one can construct both believable-invalid problems and unbelievable-valid problems). When we examined both factors separately in Experiment 2, we observed that the conflict findings were less clear for the unbelievable problems. Unbelievable material gave rise to an overall SCR boost that might have blurred an additional effect of logical validity. It is interesting to note that the conflict in other reasoning tasks typically involves a conflict between believable intuitive material (e.g., information that fits with stored stereotypes; see Kahneman \& Frederick, 2005) and normative logical or probabilistic considerations. Given the possible distorting impact of unbelievable material, this could imply that the presently observed conflict-related autonomic response might be even clearer in nonsyllogistic reasoning tasks. This underscores the importance of generalizing our findings in future studies. Overall, it must be remembered that the present study was only the first to look for possible conflict-related autonomic arousal during thinking. Clearly, this pioneering status also implies that, in the absence of future replication, the findings need to be interpreted with caution.

Another issue that will need further study is the precise conceptualization of the observed conflict-related autonomic arousal. We noted that the presence of an autonomic conflict response supports the idea that conflict detection can be conceived as a "gut" conflict feeling, and that the bias is detected at an implicit level. It should be clear that our use of the label implicit here refers to the well-established fact that biased reasoners do not explicitly refer to any violation of logical principles when asked for a verbal response justification. Hence, the crucial logical knowledge that must be present in order to detect a conflict with one's intuitive beliefs has been characterized as implicit knowledge. Studies that started focusing on conflict detection during thinking have, therefore, typically referred to the detection as an implicit process (e.g., De Neys \& Franssens, 2009; De Neys \& Glumicic, 2008; Franssens \& De Neys, 2009). However, this does not imply that the arousal generated by the con- 
flict is also implicit, in the sense that it is not consciously experienced. We do believe that people are perceiving the arousal consciously, and that this arousal informs them that their judgment is questionable; our point is simply that people will not manage to explain why their judgment is logically questionable. Note that the idea that people consciously experience the outcome of more implicit processing is quite generally accepted within the reasoning field (e.g., Evans, 2008). Consistent with this idea, recent work by our group showed that, although reasoners do not explicitly mention logical principles, they are indicating that they doubt their response; this is shown, for example, by decreased confidence ratings for biased conflict responses (De Neys, Cromheeke, \& Osman, 2009). At the same time, however, we need to acknowledge that the mere idea of an unconscious conflict feeling has not been given serious consideration in the research on conflict detection during thinking. Recent work on unconscious feelings and levels of awareness in intuitive judgments (e.g., Topolinski \& Strack, 2009; Winkielman \& Berridge, 2004) indicates that the possibility of unconscious autonomic arousal cannot be a priori excluded. An interesting idea to address this issue more directly in further studies would be to specifically ask people to report any experienced arousal. Hence - although we do believe that the conflict-related arousal feeling is consciously perceived - it will be clear that this claim needs to be further validated. On the basis of the present findings per se, the safest course of action would be to refrain from drawing strong conclusions about the ultimate experiential status of the observed conflict arousal.

Although we acknowledge that the study of the conflict detection process during reasoning is still in its infancy, we do want to point to some possible practical implications of the findings. We have argued previously that specifying the nature of the intuitive bias is crucial to designing more effective intervention strategies to debias and improve human reasoning and decision making (De Neys et al., 2008). Intuitively, the bias-as-detectionfailure view is very appealing. Most people seem to assume that giving a biased response implies that one does not know the correct answer. This is reflected in the design of experimental intervention studies, in which participants are typically tutored about logic or probability theory. Broader sociological and governmental intervention attempts often share this implicit providethe-knowledge idea: As a society, we spend billions of dollars on campaigns to inform the public about the dangers of anything from smoking to drug use to obesity. Unfortunately, these interventions have typically not been very effective (e.g., Hornik, Jacobsohn, Orwin, Piesse, \& Kalton, 2008; Kahneman, Slovic, \& Tversky, 1982; Paek \& Gunther, 2007). Given our findings, this failure is not necessarily surprising. Bluntly put, if people already detect the unwarranted nature of their intuitive decisions, it is clear that simply telling them "smoking kills" or "fast food equals fat food" is not going to have much of an impact on their decision to light another cigarette or order a second Big Mac. Although we realize that caution is needed when generalizing from studies with formal laboratory-based reasoning tasks, our findings suggest that these interventions have been targeting the wrong component of the thinking process. Future programs might be more effective if they tried to help people sidestep bad intuitions (e.g., strengthening people's inhibitory skills; see Houdé, 2007) rather than merely inform them that the intuitions are bad per se. In general, the available conflict detection evidence indicates that people are less ignorant than their biased judgments suggest.

\section{AUTHOR NOTE}

Correspondence concerning this article should be addressed to W. De Neys, Laboratoire CLLE, Maison de la Recherche, 5 allée A. Machado, 31058 Toulouse Cedex 9, France (e-mail: wim.deneys@univ-tlse2.fr).

\section{REFERENCES}

Alexander, B. (2009). Amid swine flu outbreak, racism goes viral. NBC Los Angeles. Retrieved June 11, 2009, from www.msnbc.msn .com/id/30467300/.

Ballantyne, C. (2009). Will Egypt's plans to kill pigs protect it from swine-sorry, H1N1 flu? Retrieved June 16, 2009, from www .scientificamerican.com/blog/60-second-science/post.cfm?id=will -egypts-plans-to-kill-pigs-prot-2009-05-01.

Bechara, A., Damasio, H., Tranel, D., \& Damasio, A. R. (1997). Deciding advantageously before knowing the advantageous strategy. Science, 275, 1293-1295.

Botvinick, M. M., Cohen, J. D., \& Carter, C. S. (2004). Conflict monitoring and anterior cingulate cortex: An update. Trends in Cognitive Sciences, 8, 539-546.

Botvinick, M. M., \& Rosen, Z. B. (2009). Anticipation of cognitive demand during decision-making. Psychological Research, 73, 835-842.

Critchley, H. D., Tang, J., Glaser, D., Butterworth, B., \& Dolan, R. J. (2005). Anterior cingulate activity during error and autonomic response. Neurolmage, 27, 885-895.

Damasio, A. R. (1994). Descartes' error: Emotion, reason, and the human brain. New York: Putnam.

Dawson, M. E., Schell, A. M., \& Filion, D. L. (2000). The electrodermal system. In J. T. Cacioppo, L. G. Tassinary, \& G. G. Berntson (Eds.), Handbook of psychophysiology (2nd ed., pp. 200-223). New York: Cambridge University Press.

DE NEYs, W. (2006). Dual processing in reasoning: Two systems but one reasoner. Psychological Science, 17, 428-433.

De Neys, W., Cromheeke, S., \& Osman, M. (2009). Biased but in doubt: Conflict and decision confidence. Manuscript submitted for publication.

De Neys, W., \& Franssens, S. (2009). Belief inhibition during thinking: Not always winning but at least taking part. Cognition, 113, 45-61.

De Neys, W., \& Glumicic, T. (2008). Conflict monitoring in dual process theories of thinking. Cognition, 106, 1248-1299.

De Neys, W., Schaeken, W., \& D'Ydewalle, G. (2005). Working memory and everyday conditional reasoning: Retrieval and inhibition of stored counterexamples. Thinking \& Reasoning, 11, 349-381.

De Neys, W., Vartanian, O., \& Goel, V. (2008). Smarter than we think: When our brains detect that we are biased. Psychological Science, 19, 483-489.

EPSTEIN, S. (1994). Integration of the cognitive and the psychodynamic unconscious. American Psychologist, 49, 709-724.

Evans, J. ST. B. T. (2003). In two minds: Dual-process accounts of reasoning. Trends in Cognitive Sciences, 7, 454-459.

Evans, J. ST. B. T. (2008). Dual-processing accounts of reasoning, judgment and social cognition. Annual Review of Psychology, 59, 255-278.

Evans, J. St. B. T., \& Over, D. E. (1996). Rationality and reasoning. Hove, U.K.: Psychology Press.

Franssens, S., \& De NeYs, W. (2009). The effortless nature of conflict detection during thinking. Thinking \& Reasoning, 15, 105-128.

Goel, V., \& Dolan, R. J. (2003). Explaining modulation of reasoning by belief. Cognition, 87, B11-B22.

Hajcak, G., McDonald, N., \& Simons, R. F. (2003). To err is autonomic: Error-related brain potentials, ANS activity, and post-error compensatory behavior. Psychophysiology, 40, 895-903. 
Hornik, R., Jacobsohn, L., Orwin, R., Piesse, A., \& Kalton, G. (2008). Effects of the National Youth Anti-Drug Media Campaign on youths. American Journal of Public Health, 98, 2229-2236.

HoudÉ, O. (2007). First insights on "neuropedagogy of reasoning." Thinking \& Reasoning, 13, 81-89.

Kahneman, D. (2002, December). Maps of bounded rationality: A perspective on intuitive judgment and choice. Nobel Prize lecture. Retrieved January 11, 2006, from http://nobelprize.org/nobel_prizes/ economics/laureates/2002/kahnemann-lecture.pdf.

Kahneman, D., \& Frederick, S. (2005). A model of heuristic judgment. In K. J. Holyoak \& R. G. Morrison (Eds.), The Cambridge handbook of thinking and reasoning (pp. 267-293). New York: Cambridge University Press.

Kahneman, D., Slovic, P., \& Tversky, A. (Eds.) (1982). Judgment under uncertainty: Heuristics and biases. Cambridge: Cambridge University Press.

Kobayashi, N., Yoshino, A., Takahashi, Y., \& Nomura, S. (2007). Autonomic arousal in cognitive conflict resolution. Autonomic Neuroscience, 132, 70-75.

Markovits, H., \& Nantel, G. (1989). The belief-bias effect in the production and evaluation of logical conclusions. Memory \& Cognition, 17, 11-17.

OAKhill, J., Johnson-Laird, P. N., \& Garnham, A. (1989). Believability and syllogistic reasoning. Cognition, 31, 117-140.

Paek, H.-J., \& Gunther, A. C. (2007). How peer proximity moderates indirect media influence on adolescent smoking. Communication Research, 34, 407-432.

Ridderinkhof, K. R., Ullsperger, M., Crone, E. A., \& NieuwenHUIS, S. (2004). The role of the medial frontal cortex in cognitive control. Science, 306, 443-447.

Sá, W. C., West, R. F., \& Stanovich, K. E. (1999). The domain specificity and generality of belief bias: Searching for a generalizable critical thinking skill. Journal of Educational Psychology, 91, 497-510.

Sloman, S. A. (1996). The empirical case for two systems of reasoning. Psychological Bulletin, 119, 3-22.

Stanovich, K. E., \& WeSt, R. F. (2000). Individual differences in rea- soning: Implications for the rationality debate? Behavioral \& Brain Sciences, 23, 645-726.

Thompson, V. A. (2009). Dual process theories: A metacognitive perspective. In J. St. B. T. Evans \& K. Frankish (Eds.), In two minds: Dual processes and beyond (pp. 171-195). New York: Oxford University Press.

Topolinski, S., \& Strack, F. (2009). Scanning the "Fringe" of consciousness: What is felt and what is not felt in intuitions about semantic coherence. Consciousness \& Cognition, 18, 608-618.

TsujiI, T., \& Watanabe, S. (2009). Neural correlates of dual-task effect on belief-bias syllogistic reasoning: A near-infrared spectroscopy study. Brain Research, 1287, 118-125.

van VeEn, V., \& CARTER, C. S. (2006). Conflict and cognitive control in the brain. Current Directions in Psychological Science, 15, 237-240.

Wason, P. C., \& Evans, J. St. B. T. (1975). Dual processes in reasoning? Cognition, 3, 141-154.

Winkielman, P., \& Berridge, K. C. (2004). Unconscious emotion. Current Directions in Psychological Science, 13, 120-123.

\section{NOTES}

1. A secondary analysis measuring SCR as the difference between the average skin conductance values during the reading and reasoning phases yielded comparable results.

2. In addition, a correlational analysis on the original data established that there was no association between an individual's reasoning time on the conflict problems $(r=.11, p=.53)$ or no-conflict problems $(r=$ $-.10, p=.57)$ and the observed SCR increase.

\section{SUPPLEMENTAL MATERIALS}

A detailed discussion of the materials for this article may be downloaded from http://cabn.psychonomic-journals.org/content/supplemental.

(Manuscript received July 30, 2009; revision accepted for publication December 7, 2009.) 